\title{
Spectroscopic studies of the mechanism for hydrogen-induced exfoliation of InP
}

\author{
A. Fontcuberta i Morral, ${ }^{1,2}$ J. M. Zahler, ${ }^{1}$ M. J. Griggs, ${ }^{1}$ Harry A. Atwater, ${ }^{1}$ and Y. J. Chabal ${ }^{3}$ \\ ${ }^{1}$ Thomas J. Watson Laboratory of Applied Physics, California Institute of Technology, Pasadena, California 91125, USA \\ ${ }^{2}$ Laboratoire de Physique des Interfaces et Couches Minces, Ecole Polytechnique, 91128 Palaiseau Cedex, France \\ ${ }^{3}$ Laboratory for Surface Modification, Rutgers University, Piscataway, New Jersey 08854, USA \\ (Received 4 March 2005; revised manuscript received 27 May 2005; published 24 August 2005)
}

\begin{abstract}
The motion and bonding configurations of hydrogen in InP are studied after proton implantation and subsequent annealing, using Fourier transform infrared (FTIR) spectroscopy. It is demonstrated that, as implanted, hydrogen is distributed predominantly in isolated pointlike configurations with a smaller concentration of extended defects with uncompensated dangling bonds. During annealing, the bonded hydrogen is released from point defects and is recaptured at the peak of the distribution by free internal surfaces in di-hydride configurations. At higher temperatures, immediately preceding exfoliation, rearrangement processes lead to the formation of hydrogen clusters and molecules. Reported results demonstrate that the exfoliation dynamics of hydrogen in $\mathrm{InP}$ and $\mathrm{Si}$ are markedly different, due to the higher mobility of hydrogen in InP and different implant-defect characteristics, leading to fundamental differences in the chemical mechanism for exfoliation.
\end{abstract}

DOI: 10.1103/PhysRevB.72.085219

PACS number(s): 61.72.Qq, 81.05.Ea, 78.30.-j, 52.70.Kz

\section{INTRODUCTION}

The chemical and electrical activity of hydrogen in semiconductors is of interest for a variety of fundamental and applied studies. This is especially true for hydrogen in III-V compound semiconductors due to its incorporation during the epitaxial growth of III-V device structures. Hydrogen can be incorporated as an impurity or intentionally introduced in the material to passivate the electrical activity of defects and other impurities. ${ }^{1}$ More recently hydrogen has found another utility in the field of semiconductors. Indeed, the use of hydrogen-induced exfoliation of silicon combined with wafer-bonding techniques has been applied to the layer transfer of thin silicon films onto oxidized silicon substrates ${ }^{2}$ and is the basis of the industrial production of silicon-oninsulator $(\mathrm{SOI})^{3}$ substrates. Wafer bonding now also involves semiconductors other than silicon such as $\mathrm{Ge}^{4}$ and GaAs. ${ }^{5}$ However, the H-induced exfoliation of III-V semiconductors for layer transfer onto foreign substrates has proven to be more difficult than exfoliation of Si for fabrication of SOI. Indeed, it was only recently shown that the concept of hydrogen-induced exfoliation can be applied to the transfer of thin films of InP and GaAs to foreign substrates. ${ }^{6-8}$ Moreover, a detailed understanding of the role of $\mathrm{H}$ in these materials is still lacking.

The role of hydrogen in H-induced exfoliation of silicon has been extensively studied. Several techniques have been applied to understanding the formation of hydrogen platelets and internal cavities leading to the exfoliation of a full thin film. In the delineation of a detailed model that explains the exfoliation process of silicon, Fourier transform infrared (FTIR) spectroscopy combined with other structural measurements such as transmission electron microscopy (TEM), atomic force microscopy (AFM), and forward-recoil scattering (FRS) has played a central role. ${ }^{9-11}$ In these studies, it was shown that $\mathrm{H}$-terminated vacancies form internal structures passivated with silicon mono-hydrides, where $\mathrm{H}_{2}$ gas is segregated from the bulk semiconductor, providing the inter- nal pressure necessary to form extended surfaces and cracks. The accumulation of $\mathrm{H}_{2}$ into these cavities causes the internal pressure to rise to a level where the whole surface of silicon blisters. When bonded to a handle substrate, the handle substrate acts as a stiffener, limiting the threedimensional growth of blisters and leading to exfoliation and layer transfer of a continuous film onto the handle substrate.

An understanding of the chemical and physical mechanism of $\mathrm{H}$-induced exfoliation in InP cannot be deduced from previous studies in $\mathrm{Si}$ for several reasons. First, In and $\mathrm{P}$ have different valence electronic structures, which necessarily leads to different interactions with H. Second, these two elements have two very different masses (a factor of 3.7), leading to an asymmetry number of vacancies for each element during implantation. As a consequence, implantation of InP creates more disorder and damage in the material, and can inhibit the complete healing of the structure by thermal annealing. A consequence of extreme implantation-induced disorder for the H-induced exfoliation of InP is a lack of the crystallographic planes that are required for the formation of platelets and subsequent opening of micro-cracks necessary for the exfoliation process.

In contrast with silicon, only a few spectroscopy studies of hydrogen in InP exist in the literature. Prior work has focused on the study of the vibrational modes of hydrogen bonded to external surfaces of InP where hydrogen is adsorbed to well-characterized low-index crystallographic InP surfaces, such as the (100), (110), and (111) surfaces, while other studies have investigated the so-called local vibrational modes (LVM's) of H-implanted InP in which hydrogen is opportunistically incorporated in a perturbed lattice, with relatively unknown crystal structure. In our case, the study of InP implanted under conditions leading to layer exfoliation can be considered a combination of these two conditions. On the one hand, implantation creates isolated lattice defects that lead to defect modes attributed to LVM's. On the other hand, large implantation 
doses causing large densities of defects and introducing large quantities of hydrogen should lead to the formation of internal surfaces where the hydrogen accumulates prior to the layer exfoliation.

In this paper we present a study of the effect of isochronal annealing on the vibrational modes of $\mathrm{H}$-implanted InP for the understanding of H-induced exfoliation of InP. The experimental results are discussed relative to existing microscopic models for the H-containing defect centers produced by implantation and also relative to previous studies of the H-passivation of (100), (110), and (111) InP-free surfaces. The FTIR results are complemented by hydrogen-thermalevolution measurements.

The paper is structured as follows: In Sec. II the experimental details are given. Hydrogen-thermal-evolution and FTIR measurements are then presented in Sec. III. These results along with complementary MIT-FTIR measurements are discussed in Sec. IV, to arrive at a model for the role of hydrogen in the H-induced layer exfoliation of InP, with conclusions presented in Sec. V.

\section{EXPERIMENTAL DETAILS}

The InP wafers used in this study are double-sided, polished, 50-mm undoped (100) substrates. The wafers were implanted at room temperature with $80 \mathrm{keV} \mathrm{H}^{+}$to a dose of $10^{17} \mathrm{~cm}^{-2}$, then cleaved into individual samples measuring $15 \mathrm{~mm} \times 40 \mathrm{~mm}$ for simple transmission and multiple internal transmission (MIT) measurement configurations. ${ }^{12}$ The MIT samples were beveled at $45^{\circ}$. At the bevels, light enters by normal incidence from the medium of low refractive index to the medium of high refractive index (from air to InP). At the first reflection with the bottom of the InP prism, the angle is such that the light is totally reflected at the interface. The light is again totally reflected at the top of the prism and therefore reflected many times inside the sample. At each reflection, the light is transmitted through the region of interest (the implanted InP); this is why the configuration is called multiple internal transmission.

All InP pieces were annealed isochronally in a nitrogen ambient. For transmission measurements, the same sample holder was equipped with a calibrated resistance heater, allowing an in situ annealing inside a nitrogen-purged chamber located in the spectrometer. The sample was therefore not exposed to air between isochronal annealing steps and the measurements. Annealing in situ also guaranteed that the $45^{\circ}$ angle of the sample relative to the IR beam and detector was not perturbed.

The hydrogen-evolution measurement was performed as follows: a $1 \mathrm{~cm}^{2} \mathrm{InP}$ sample was placed inside a vacuum furnace equipped with a mass spectrometer with a base pressure of $10^{-9}$ Torr. The InP was then annealed up to $340{ }^{\circ} \mathrm{C}$ at a rate of $10^{\circ} \mathrm{C}$ per minute, and the partial pressure of $\mathrm{H}_{2}$ was measured by the mass spectrometer. The temperature in the vacuum furnace for the hydrogen evolution, the FTIR sample heater, and the furnace were all calibrated using a silicon thermocouple-instrumented wafer from Sensarray, which has an absolute temperature accuracy of $1.1{ }^{\circ} \mathrm{C}$. This calibration enables a fair comparison of the temperature-dependent behavior measured by the different techniques.

\section{RESULTS}

Hydrogen-implanted InP exhibits multiple infrared absorption peaks and bands at the infrared, as illustrated in Figs. 1(a) and 2. Some of these bands and peaks are attributed to In-H and P-H stretching modes associated with both discrete defects and H-passivated internal surfaces. Transmission FTIR spectra were measured from the as-implanted sample and following 10-minute isochronal annealing in $60{ }^{\circ} \mathrm{C}$ increments from $112{ }^{\circ} \mathrm{C}$ to $412{ }^{\circ} \mathrm{C}$. All measurements were taken at $50{ }^{\circ} \mathrm{C}$. In- $\mathrm{H}, \mathrm{P}-\mathrm{H}$, and surfaceadsorbed water and related modes have different relative intensities and are located in different parts of the spectrum. To better illustrate the different infrared features observed, the full spectra were divided into three graphs showing the In- $\mathrm{H}$ modes from 1250 to $2000 \mathrm{~cm}^{-1}$ and the $\mathrm{P}-\mathrm{H}$ modes from 2100 to $2500 \mathrm{~cm}^{-1}$ in Figs. 1(a) and 2, respectively, and the adsorbed water modes from 3000 to $4000 \mathrm{~cm}^{-1}$ in Fig. 1(b).

Bands centered at 1600 and $1705 \mathrm{~cm}^{-1}$ illustrated in Fig. 1(a) are associated with the vibration of $\mathrm{In}-\mathrm{H}$ modes, specifically to terminal In hydrides. ${ }^{13-15}$ These modes are slightly shifted from the modes found on In-terminated surfaces. We attribute this shift to the different environment where the $\mathrm{H}$ is located. However, theoretical calculations should be done to state this with more certainty and to understand the origin of the shift as well as to attribute the bands to the symmetric and asymmetric modes. The large width of the bands and their annealing behavior strongly suggests that $\mathrm{In}-\mathrm{H}$ modes are not located in well-defined planes but could consist of point defects, such as hydrogendecorated phosphorus vacancies. At room temperature, these two bands seem to be merged into a single, broad band. We attribute this lack of separation to the presence of a third band at $1650 \mathrm{~cm}^{-1}$. This third band corresponds to the presence of water molecules adsorbed at the surface of the sample, attributed to the $\mathrm{H}-\mathrm{O}-\mathrm{H}$ scissor mode, and it is well correlated with the intensity of the $\mathrm{H}-\mathrm{O}-\mathrm{H}$ stretch modes of adsorbed water observed at $3500 \mathrm{~cm}^{-1}$, as shown in Fig. 1(b). The adsorption of water at the surface is a consequence of the InP surface hydrophilicity. Indeed, it has been shown that InP surfaces adsorb several monolayers of water following a brief exposure to air, even if $\mathrm{H}$-passivated by being treated in HF. ${ }^{16,17}$ As illustrated in Fig. 1(b), all spectral bands associated with water disappear after annealing to $292{ }^{\circ} \mathrm{C}$, indicating that the water has been thermally desorbed from the surface or converted to an $\mathrm{InPO}_{x}$ surface layer. Following the loss of water at the InP surface at $292^{\circ} \mathrm{C}$, the bands attributed to the $\mathrm{In}-\mathrm{H}$ modes become clearer and have a reduced spectral overlap.

The In-H bands centered at 1600 and $1704 \mathrm{~cm}^{-1}$ exhibit uncorrelated annealing behavior. While the intensity of the band at $1600 \mathrm{~cm}^{-1}$ remains nearly unaffected during annealing, the band at $1704 \mathrm{~cm}^{-1}$ decreases in intensity by $27 \%$. In both cases the bands become narrower upon annealing, but it 

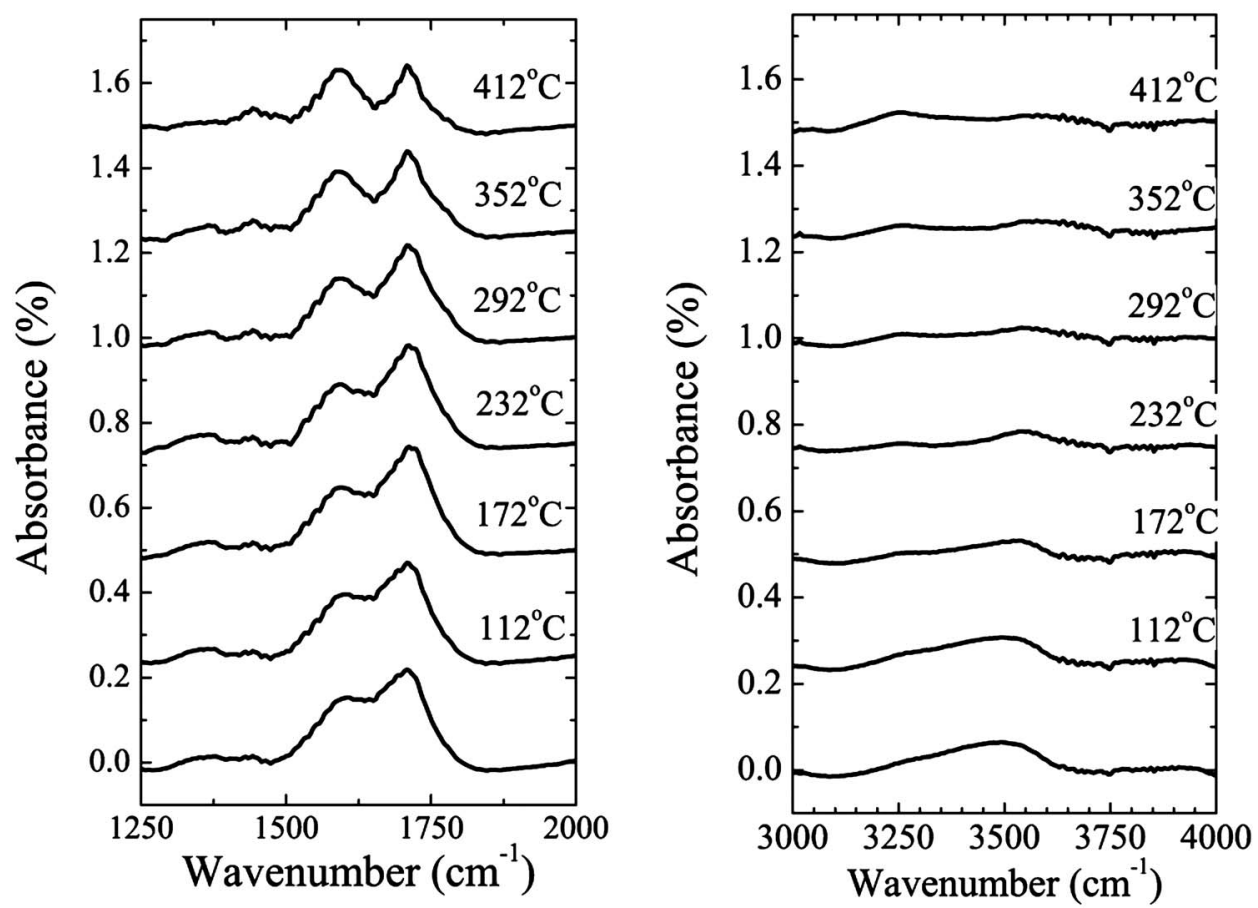

FIG. 1. Transmission FTIR spectra around (a) $1600 \mathrm{~cm}^{-1}$ and (b) $3500 \mathrm{~cm}^{-1}$, corresponding to In-H and adsorbed water modes. The bottom spectrum corresponds to as-implanted InP, and the rest of the spectra to the same InP annealed at different temperatures for 10 minutes. Spectra are displayed vertically for comparison purposes.

is difficult to make a quantitative analysis, due to the presence of the $\mathrm{H}-\mathrm{O}-\mathrm{H}$ scissor-mode band at $1650 \mathrm{~cm}^{-1}$ at lower temperatures. In general, it appears that In-H complexes in implanted $\mathrm{InP}$ are stable relative to $\mathrm{P}-\mathrm{H}$ up to $412{ }^{\circ} \mathrm{C}$. It has been found that the insertion of hydrogen into metal dimer bonds releases a substantial amount of strain in the In-P back-bonds. ${ }^{14}$ The enthalpy of formation of this process is $+52.2 \mathrm{kcal} \mathrm{mol}^{-1}$, corresponding to a bondformation energy of $2.6 \mathrm{eV},{ }^{18}$ meaning that the final state is more stable. The stress release and large bond-formation energy are two possible origins of the observed thermal stability of the In-H bonding configurations. This observation has important implications for the exfoliation of InP. Specifically, the relative difficulty of performing the $\mathrm{H}$-induced exfoliation process in InP, as compared to $\mathrm{Si}$, can be partially explained by the sequestering of hydrogen at anion vacancies. Once In- $\mathrm{H}$ bonds are formed at these anion vacancies, the bonded $\mathrm{H}$ is no longer free to diffuse to extended defect structures, where it can contribute to internal pressure that leads to exfoliation.

The evolution of $\mathrm{P}-\mathrm{H}$ infrared spectra under isochronal annealing is shown in Fig. 2. The spectrum of the $50{ }^{\circ} \mathrm{C}$ sample is composed of two clear peaks at $2306 \mathrm{~cm}^{-1}$ and $2198 \mathrm{~cm}^{-1}$ punctuated by a series of overlapping peaks at intermediate frequencies, specifically at 2217, 2227, 2268, and $2275 \mathrm{~cm}^{-1}$. All of these peaks are associated with $\mathrm{P}-\mathrm{H}$ modes that will be identified and discussed in the next section with the aid of higher-resolution MIT-mode spectra. Here a brief description of the evolution of the $\mathrm{P}-\mathrm{H}$ during sequential isochronal annealings is presented. There is no change in the spectrum after annealing the sample for 10 minutes at $112{ }^{\circ} \mathrm{C}$. After annealing at $172{ }^{\circ} \mathrm{C}$ the over-

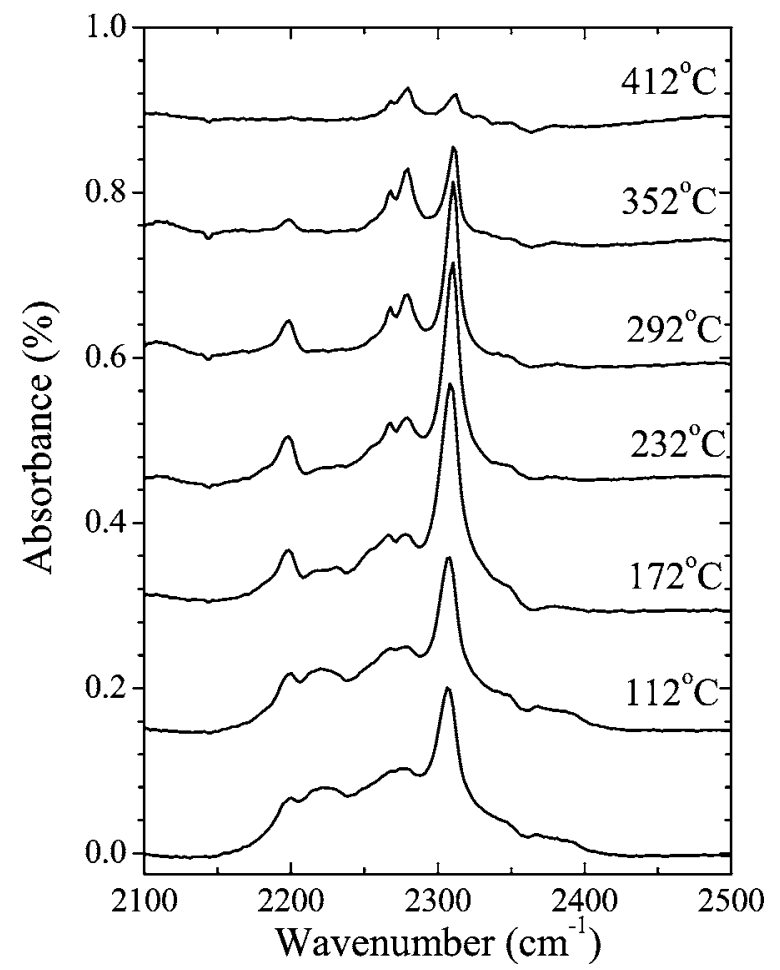

FIG. 2. Transmission FTIR spectra around the region where $\mathrm{P}-\mathrm{H}$ modes absorb. The bottom spectrum corresponds to implanted InP, and the rest of the spectra to the same InP annealed at different temperatures for 10 minutes. Spectra are displayed vertically for comparison purposes. 


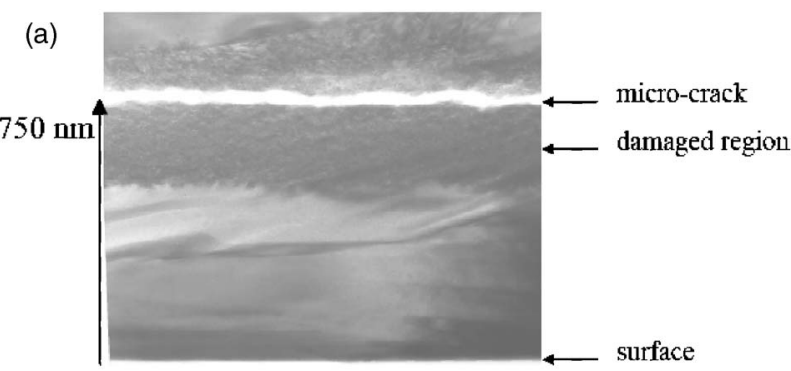

(b)

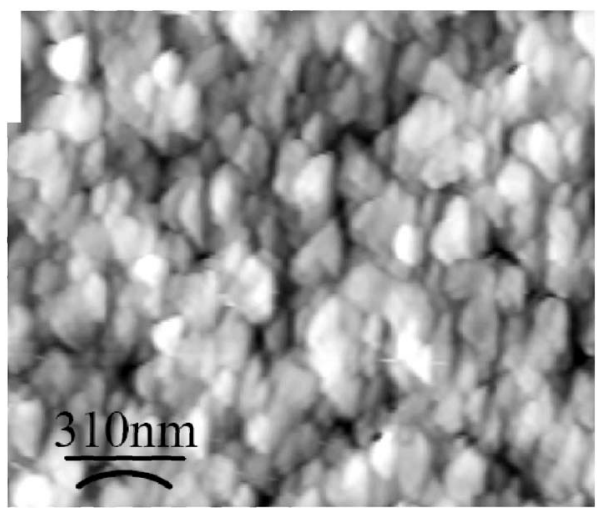

FIG. 3. (a) Cross-section TEM micrograph of the implanted InP, showing extended defects parallel to the (100) plane, and (b) AFM measurement of the surface of an exfoliated InP showing the (100) cleavage planes as (100) oriented mesas.

lapping peaks between 2217 and $2227 \mathrm{~cm}^{-1}$ begin to decrease in intensity, disappearing completely after annealing to $292{ }^{\circ} \mathrm{C}$. The remaining peaks generally sharpen as the annealing proceeds, while each peak exhibits a unique evolution upon annealing. The intensities of the peaks at 2198 , 2268 , and $2275 \mathrm{~cm}^{-1}$ decrease, with the peak at $2198 \mathrm{~cm}^{-1}$ nearly disappearing by $352{ }^{\circ} \mathrm{C}$, while the peaks at 2268 and $2278 \mathrm{~cm}^{-1}$ are still observed after annealing to $412{ }^{\circ} \mathrm{C}$. In addition, the position of the peaks at 2198, 2268, and $2275 \mathrm{~cm}^{-1}$ does not change, while the position of the peak at $2306 \mathrm{~cm}^{-1}$ is shifted to higher energy by $6 \mathrm{~cm}^{-1}$, during which its intensity first increases, reaching a maximum at $232{ }^{\circ} \mathrm{C}$, and subsequently decreases significantly by the $412{ }^{\circ} \mathrm{C}$ annealing. In comparison to previous work and as will be shown in the next section, it is determined that the lower-frequency modes correspond to isolated H-passivated defects, whereas the higher-frequency modes correspond to hydrogen complexes.

From these measurements several conclusions can be drawn. In the range from $50{ }^{\circ} \mathrm{C}$ to $292{ }^{\circ} \mathrm{C}$, the simultaneous increase of absorption in the higher-wave-number modes and decrease of absorption in the lower-wave-number modes suggests that upon annealing in this temperature range hydrogen bonded to point defects is thermally released from these structures and populates extended defects that it reaches during diffusion. At higher temperatures, the highfrequency modes decrease in intensity, indicating that a fraction of the hydrogen-forming di-hydrides is debonded. Finally, at temperatures higher than $350{ }^{\circ} \mathrm{C}$, InP blisters, most of the mono- and di-hydrides have been decomposed, and very little hydrogen is left in the material.

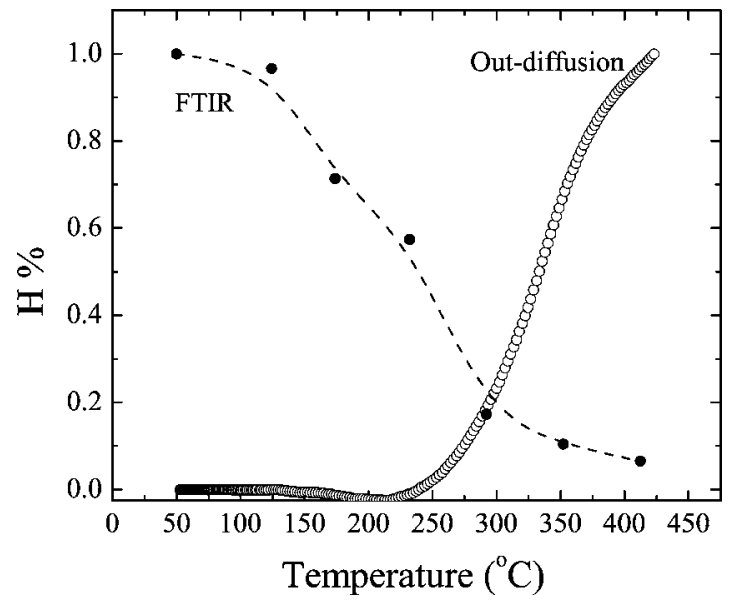

FIG. 4. Illustration of the fraction of hydrogen evolved as a function of temperature deduced from FTIR.

In Fig. 3 structural characterization of the exfoliation process in InP is presented. In Fig. 3(a) a cross-section TEM micrograph reveals the presence of extended cracks along the implanted region after annealing at greater than $350{ }^{\circ} \mathrm{C}$. As shown in the micrograph, the cracks open and extend parallel to the (100) surface, indicating that the exfoliation occurs in the peak of the implanted-hydrogen distribution. This observation is further corroborated by an AFM image of the blistered surface shown in Fig 3(b), where the presence of (100) parallel mesas are clearly present, further indicating that cleavage proceeds along the (100) direction during exfoliation of InP.

In Fig. 4, the total area under the $\mathrm{P}-\mathrm{H}$ bands as a function of the annealing temperature is also indicated. The area has been normalized to the total area under the spectra before annealing, and tends to indicate the fraction of hydrogen bonded to $\mathrm{P}$ in the material. After annealing at $232{ }^{\circ} \mathrm{C}$ about $30 \%$ of hydrogen is lost from $\mathrm{P}-\mathrm{H}_{x}$ modes prior to its loss from the bulk InP material, which we attribute to the formation and trapping of $\mathrm{H}_{2}$ clusters and molecules. At $300{ }^{\circ} \mathrm{C}$, only $30 \%$ of the initially bound hydrogen is still remaining. The proportion of bound hydrogen continues to decrease after annealing at higher temperatures. After annealing at $412{ }^{\circ} \mathrm{C}$, InP has blistered and very little hydrogen is left in the material. To complement the information given by FTIR, the evolution of hydrogen from the InP bulk was measured in a vacuum furnace with a mass spectrometer tuned to mass 2 , in order to assess the loss of hydrogen in the material during the annealing cycle. Only after reaching a temperature of $300{ }^{\circ} \mathrm{C}$ is a significant amount of hydrogen diffusing out of the InP detected. The diffusion of hydrogen out of the InP is very rapid and ends at $350{ }^{\circ} \mathrm{C}$ during exfoliation. This observation is in agreement with the interpretation that hydrogen released from discrete defect structures diffuses in the bulk, where a significant quantity of the mobile hydrogen is captured by extended defects and contributes to internal pressure leading to exfoliation.

\section{DISCUSSION}

Hydrogen forms a relatively strong chemical bond with In and P. However, the bond strength must depend on the sur- 
rounding environment and the type of defect in which the hydrogen is bound. Moreover, the evolution of the bonds is kinetically limited, because it is easier for $\mathrm{H}$ to leave from a larger position such as an In vacancy. H-covered InP-free surfaces are ideal for research of chemisorption processes at semiconductor surfaces. ${ }^{13,19,20}$ However, the geometry and crystallography do not correspond well to our experimental situation. Indeed, even if in $\mathrm{H}$-induced exfoliation processes the hydrogen is supposed to evolve by forming H-stabilized internal surfaces, the configuration and bonding of hydrogen to those internal surfaces is strongly influenced by the interaction of these In-H and P-H surface modes with similar modes on opposing surfaces in micro-cracks. Additionally, strain-induced vibrational shifts caused by local damage in the InP or by high-pressure $\mathrm{H}_{2}$ molecules can cause significant shifting of surface modes in InP micro-cracks from the measured values on ideal InP external surfaces. Moreover, it is expected that implantation will displace some In and P atoms, creating dangling bonds and damaging the structure. As the immediate surrounding is different from free surfaces, it is expected that the frequencies of hydrogen located in internal cavities and in defect structures created by implantation will be shifted and broadened relative to measurements made on free surfaces. Finally, hydrogen modes should also exhibit different annealing behavior and a different polarization response, depending on their location and surrounding environment.

In this section, we present MIT-FTIR experimental results for the purpose of better elucidating the chemical states of hydrogen in H-implanted InP. MIT-mode FTIR spectroscopy makes this possible because of the greater signal-to-noise performance enabled by the enhancement that occurs when the IR beam makes multiple passes through the absorbing medium. In the MIT-FTIR configuration light is introduced through one bevel at the end of the prism sample and makes approximately 57 passes through the sample prior to exiting the opposite bevel and being directed to the detector. As a consequence of being a multiple-pass experiment, MIT measurements are more sensitive than single-pass transmission, making it easier to resolve weak spectral features. The geometry is denoted MIT since the incident light is able in each reflection to tunnel through the implanted region, which is much thinner than the wavelength of the radiation of interest. ${ }^{21}$ Additionally, by polarizing the IR beam prior to entry into the MIT prism, it is possible to deduce the dipole orientation of the observed modes, thus assisting in the interpretation of the spectra. Moreover, when the implanted species are within a few wavelengths from the external surfaces, interference effects lead to strong-intensity modulation for components of the modes as a function of distance from the external surface of the prism. It is therefore possible in some cases to determine spatial information from the spectra. Specifically, the light intensity of each polarization is proportional to the square of the field components and is expressed as follows:

$$
\begin{aligned}
\left|\vec{E}_{s}\right|^{2} & =4 E_{o}^{2} \cdot\left|\overrightarrow{e_{y}} \cdot\left[\exp \left(i k_{z} \cdot z+\Delta \psi\right)+\exp \left(i k_{z} \cdot z\right)\right]\right|^{2} \\
& =4 E_{o}^{2} \cdot\left|\sin \theta \cos \left(k_{z} \cdot z+\frac{\Delta \psi}{2}\right)\right|^{2},
\end{aligned}
$$

a)
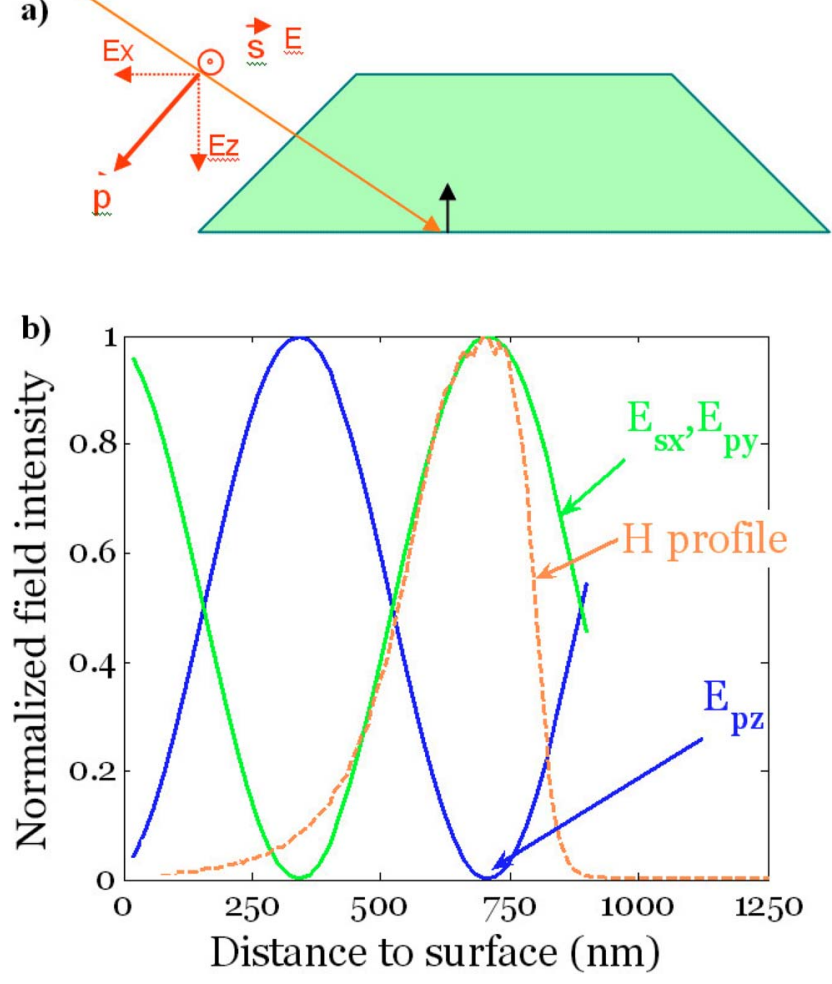

FIG. 5. (Color online) (a) Schematics of the notation of the parallel and perpendicular components of the electrical field of the radiation incident to the prism. (b) Normalized intensity of the field components as a function of the distance to the surface, overlapped with the hydrogen profile in InP after current implantation.

$$
\begin{aligned}
\left|\vec{E}_{p}\right|^{2}= & 4 E_{o}^{2} \cdot \mid \overrightarrow{e_{x}} \cdot\left[\exp \left(i k_{z} \cdot z+\Delta \psi\right)+\exp \left(i k_{z} \cdot z\right)\right] \\
& +\left.\overrightarrow{e_{z}} \cdot\left[\exp \left(i k_{z} \cdot z+\psi_{p}\right)+\exp \left(i k_{z} \cdot z\right)\right]\right|^{2}, \\
= & E_{o}^{2} \cdot\left|\sin \theta \cos \left(k_{z} \cdot z+\frac{\Delta \psi}{2}\right)\right|^{2} \\
& +E_{o}^{2} \cdot\left|\cos \theta \cos \left(k_{z} \cdot z+\frac{\psi_{p}}{2}\right)\right|^{2}, \\
\frac{\psi_{p}}{2}= & -\arctan \frac{n^{2} \sqrt{n^{2} \sin ^{2} \theta-1}}{n \cos \theta} ; \quad \frac{\Delta \psi}{2}=\frac{\pi}{2}-\frac{\psi_{p}}{2},
\end{aligned}
$$

where $E$ corresponds to the electric field, and subindexes $s$, $p, x, y, z$ correspond respectively to the components of polarization $s$ and $p$ and are parallel to the axes $x, y$, and $z . E_{o}$ is the modulus of the electric field, $\theta$ is the incidence angle, $\psi$ is the phase change after reflection on one side of the prism, and $\Delta \psi$ is the shift in the phase change due to the evanescence of the light during reflection.

The convention used for the polarization is schematically illustrated in Fig. 5(a). Polarization type $s$ corresponds to the component perpendicular to the incidence plane, whereas polarization type $p$ corresponds to the component parallel to the incidence plane. In Fig. 5(b), the field intensity of the $x, y$, and $z$ components is plotted as a function of the distance to 

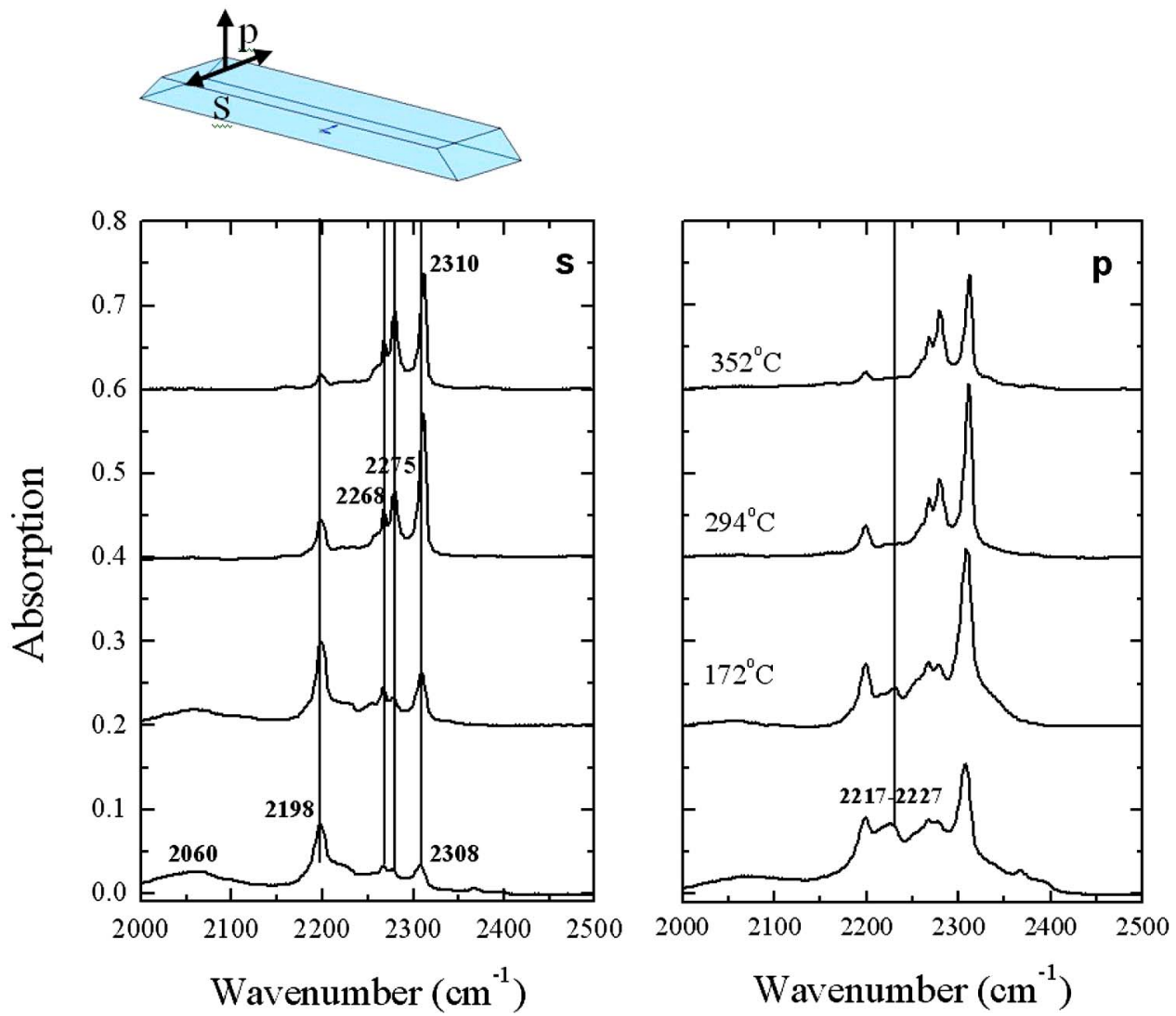

FIG. 6. (Color online) MIT-FTIR spectra of hydrogen-implanted InP as implanted and after 10 minute isochronal annealings at 172, 294, and $352^{\circ} \mathrm{C}$ for two light polarizations: (a) polarization $s$ and (b) polarization $p$. Spectra are displayed vertically for comparison purposes.

the interface. The hydrogen distribution in InP after implantation, for the implant conditions used, is also shown. The peak of the H-distribution occurs where the the $z$ component of the $p$ polarization is extinguished. As a consequence, the sensitivity to symmetric (100) P-H modes in the peak of the hydrogen implantation, where exfoliation occurs, is zero. Interestingly, at that position the $y$ component of $p$-polarized light and $s$-polarized light are equally intense. Therefore if all bound hydrogen was located at a depth of $700 \mathrm{~nm}, s$ and $p$ peak intensities should be equal. For positions closer to the external surface, the $z$ component of the $p$-polarized light increases, while the $x$ and $y$ components of the $p$ - and $s$-polarized light, respectively, decrease. Therefore, the intensity of $p$-polarized light is higher for distances closer to the surface. As a consequence of the different spatial intensity of $p$ - and $s$-polarized light, it is possible to obtain spatial information on the bound hydrogen by comparing the intensity between measurements done at different polarizations.

Before entering into detail on the consequences of the extinguished $z$ component at the H-concentration peak, the origin of the peaks observed in the 2100 to $2300 \mathrm{~cm}^{-1}$ region as measured by MIT-FTIR is discussed (Fig. 6). Figs. 6(a) and 6(b) show the absorbance spectra of InP after successive 10-minute isochronal annealing at temperatures ranging from $172{ }^{\circ} \mathrm{C}$ to $352{ }^{\circ} \mathrm{C}$. The samples were not annealed to higher (exfoliation) temperatures due to limitations in the furnace, and due to the fact that we would have lost nearly all the multiple-pass signal due to the imperfect, non-flat, blistered external surface.
A discussion of the MIT-FTIR is presented in the following paragraphs. In order to provide a clearer image, schematics of the hydrogen configurations that are associated with the different MIT-FTIR observed modes are shown in Fig. 7. They will be introduced along with the discussion.

In comparison to the single-pass transmission-mode measurements, the MIT-mode spectra are more sensitive to de-

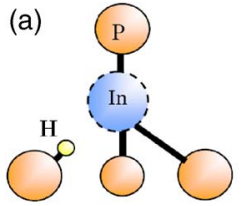

(d)

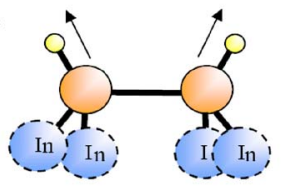

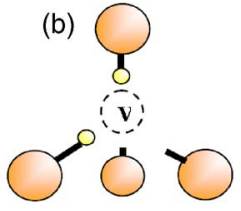

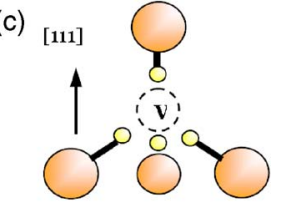

(e)
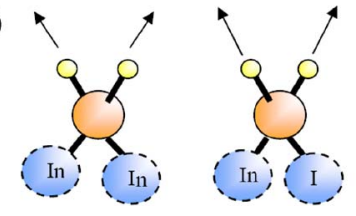

FIG. 7. (Color online) Schematics of the five different P-H bond configurations corresponding to the stretching modes of $\mathrm{H}$ in InP. Respectively, orange, blue, and yellow circles correspond to phosphorous, indium, and hydrogen atoms. From (a) to (c) the figures correspond to defect configurations (modes at $2060 \mathrm{~cm}^{-1}$, $2198 \mathrm{~cm}^{-1}$, and $2217-2227 \mathrm{~cm}^{-1}$ ). (d) and (e) correspond to stretching vibrations of mono- and di-hydrides on the (100) InP plane (modes at 2268-2275 $\mathrm{cm}^{-1}$, and $2308-2310 \mathrm{~cm}^{-1}$ ). 
fects present in small concentration. For instance, two new absorption peaks appear at $2060 \mathrm{~cm}^{-1}$ and 2250 to $2258 \mathrm{~cm}^{-1}$ in the MIT-mode measurements. While the mode at $2060 \mathrm{~cm}^{-1}$ was not observed in transmissionmode measurements described in the preceding section due to the inferior sensitivity of single-pass transmission-mode measurements, the mode from 2250 to $2258 \mathrm{~cm}^{-1}$ could not be detected because it was obscured by two adjacent peaks. After implantation, all of the modes except the modes at $2060 \mathrm{~cm}^{-1}$ and $2198 \mathrm{~cm}^{-1}$ mode exhibit slightly enhanced absorbance in $p$ polarization. Despite the identification of a mode at $2050 \mathrm{~cm}^{-1}$ in previous studies associated with the $\mathrm{P}-\mathrm{H}$ stretch of an H-passivated (111) surface, ${ }^{22}$ the disappearance of the mode at $2060 \mathrm{~cm}^{-1}$ between 172 and $294{ }^{\circ} \mathrm{C}$ indicates that it is an LVM associated with a discrete hydrogenated defect or distribution of related discrete defects having similar chemical structure, such as a hydrogenated interstitial defects [Fig. 7(a)]. This explanation is consistent with the fact that this mode was never observed in works where $\mathrm{H}$-passivated surfaces were studied. The $2198 \mathrm{~cm}^{-1}$ mode is very close to the LVM at $2206 \mathrm{~cm}^{-1}$ measured by Fischer et al. and in perfect agreement with the mode measured by Riede et al..$^{23-26}$ In both references, this mode is attributed to $\mathrm{P}-\mathrm{H}$ vibrations of a hydrogen atom localized in a cation vacancy, as depicted in Fig. 7(b). Eventually, this kind of defect could be filled with more than one hydrogen atom but, as will be presented in the following paragraph, less than four.

Modes at 2217 and $2227 \mathrm{~cm}^{-1}$ correspond to the stretch modes of $\mathrm{H}$-decorated In vacancies, $\mathrm{V}_{\mathrm{In}} \mathrm{H}_{4}$, as shown in Fig. 7(c) ${ }^{23}$ In this configuration, the four hydrogen atoms form a tetrahedron and the vibrational dipoles are oriented versus the [111] direction. The mode corresponds to the collective stretching of the four hydrogen atoms. Such vacancies are located in the region above the implant end of the range, where the $z$ component of $p$-polarized light is roughly three times as intense as the $x$ component of $s$-polarized light and the $y$ component of $p$-polarized light. This mode has been nearly annealed out at $294^{\circ} \mathrm{C}$, where the peak is still slightly present in the $\vec{p}$-polarized spectrum and completely nonexistent in the $\vec{S}$. The mode at $2268 \mathrm{~cm}^{-1}$ is close to the frequency of $2265 \mathrm{~cm}^{-1}$ attributed in previous work to symmetric stretch modes of H-terminated (100) surfaces with a $2 \times 1$ reconstruction. ${ }^{14}$ The mode corresponds to a dimer formed by two adjacent atoms, as depicted in Fig. $7(\mathrm{~d})$. The mode at $2308 \mathrm{~cm}^{-1}$ has been theoretically predicted to be the symmetric stretching vibration of a $\mathrm{P}-\mathrm{H}_{2}$ complex on a $\langle 100\rangle$ InP surface [see Fig. 7(e)]. Experimentally, this mode has been measured at $2317 \mathrm{~cm}^{-1}$ on H-passivated phosphorus-rich (001)- $(2 \times 1)$ InP surfaces. The antisymmetric pair is predicted to be found at $2332 \mathrm{~cm}^{-1}$ with lower intensity than the symmetric mode, and is not detected in our measurements. Such di-hydride complexes could be found both in cation vacancies and at internal surfaces. ${ }^{27,28}$ At the same time, this di-hydride dipole could be isotropically oriented, with equal contributions along all axes $(x, y, z)$, because it is not located on a particular surface.

We have used the relative intensity between polarizations in order to get spatial information. At the implant conditions we have chosen for this study, the maximum hydrogen concentration is located at a distance to the surface corresponding to a position where the $z$ component of the electric field is zero and therefore $p$ - and $s$-polarized spectra should have $x$ - and $y$-component electric fields with equal intensities. As indicated in Fig. 5(b), for distances closer to the external surface, the field intensity of the $s$-polarized spectrum becomes weaker in comparison to the field intensity of the $p$-polarized spectrum. Given the difference in intensity of each peak between the $p$ - and $s$-polarized spectra, the approximate location of the modes can be deduced. For instance, in the as-implanted spectra, all of the $p$-polarized peaks have a stronger intensity than the $s$-polarized ones. Only the peak present at $2198 \mathrm{~cm}^{-1}$ has the same intensity in the $s$ - and $p$-polarized spectra, which indicates that these types of defects are mainly located at a distance of $\sim 700 \mathrm{~nm}$ from the surface, where the hydrogen concentration is maximum.

As the annealing proceeds, the intensity differences between the two polarizations become less apparent, and at $352{ }^{\circ} \mathrm{C}$ they are nearly equivalent. The intensity of the peak at $2308 \mathrm{~cm}^{-1}$ increases with annealing up to $294{ }^{\circ} \mathrm{C}$, where it reaches a maximum. The fact that there is a simultaneous increase of the higher-wave-number LVM's with a decrease of the lower-wave-number LVM's along with the equilibration of the $p$ - and $s$-polarized spectra, suggests that the release of bonded hydrogen in regions between the hydrogen peak concentration and the outer surface are due to pointdefect annealing. This released hydrogen is partially captured at the free internal surfaces of voids and/or extended defect structures located at the peak of the $\mathrm{H}$-implanted distribution. Indeed, other studies have shown that the formation of clusters of cation vacancies, including di-vacancies, can be expected at sufficiently high $\mathrm{H}$-implantation doses. Of these defects, the larger defects are predominantly formed in regions of the implanted layer with high-damage densities close to the damage peak. ${ }^{29}$ The grouping of hydrogen into the internal surfaces at the peak of the distribution is analogous to a self-gettering process, and it is responsible for the collection of $\mathrm{H}_{2}$ gas that provides internal pressure and leads to blister formation and exfoliation of InP films upon annealing.

\section{CONCLUSION}

FTIR measurements of $\mathrm{H}$-implanted InP have been carried out to investigate the $\mathrm{H}$-induced exfoliation processes in InP. As implanted, the hydrogen is distributed in isolated discrete defects and extended defects with uncompensated dangling bonds. FTIR spectra provide insight into the predominant types of defects and consequently into the environments of bonded hydrogen in H-implanted InP. Additionally, polarized MIT-mode spectra shed light on the distribution of defects within the implanted region. The simultaneous occurrence of an increase of absorption in higher-wave-number local modes, attributed to agglomerated H-passivated defects, and a decrease of absorption in lower-wave-number local modes, attributed to hydrogen bonded in isolated va- 
cancies, suggests that the release of bonded hydrogen due to point-defect annealing is accompanied by its capture at the free internal surfaces of extended defects. Polarizationdependent MIT-mode spectra suggest that the desorbed atomic hydrogen from single vacancies distributed toward the outer surface of the InP substrate during annealing dif- fuses to internal surfaces and cavities located at the peak of the distribution. At higher temperatures, there is a decrease of absorption in the highest-frequency modes that is attributed to rearrangement processes that lead to trapping and buildup of $\mathrm{H}_{2}$ molecules and their associated pressure just prior to exfoliation.
${ }^{1}$ C. G. Van de Walle and J. Neugebauer, Nature (London) 423, 626 (2003).

${ }^{2}$ M. Bruel, Electron. Lett. 31, 1201 (1995).

${ }^{3}$ M. Bruel, Electron. Lett. 31, 1201 (1995).

${ }^{4}$ J. M. Zahler, C. G. Ahn, S. Zaghi, H. A. Atwater, C. Chu, and P. Iles, Thin Solid Films 403, 558 (2002).

${ }^{5}$ U. Goesele and Q. Y. Tong, Annu. Rev. Mater. Sci. 28, 215 (1998).

${ }^{6}$ A. Fontcuberta i Morral, J. M. Zahler, and H. A. Atwater, Appl. Phys. Lett. 83, 5413 (2003).

${ }^{7}$ I. Radu, I. Szafraniak, R. Scholz, M. Alexe, and U. Goesele, J. Appl. Phys. 94, 7820 (2003).

${ }^{8}$ S. Hayashi, D. Bruno, and M. S. Goorsky, Appl. Phys. Lett. 85, 236 (2004).

${ }^{9}$ M. K. Weldon, V. E. Marsico, Y. J. Chabal, D. R. Hamann, S. B. Christman, and E. E. Chaban, Surf. Sci. 368, 163 (1996).

${ }^{10}$ M. K. Weldon, M. Collot, Y. J. Chabal, V. C. Venezia, A. Agarwal, T. E. Haynes, D. J. Eaglesham, S. B. Christman, and E. E. Chaban, Appl. Phys. Lett. 73, 3721 (1998).

${ }^{11}$ Y. J. Chabal, M. K. Weldon, Y. Caudano, B. B. Stefanov, and K. Raghavachari, Physica B 274-274, 152 (1999).

${ }^{12}$ Y. J. Chabal, Surf. Sci. Rep. 8, 211 (1988).

${ }^{13}$ L. Li, B.-K. Han, Q. Fu, and R. F. Hicks, Phys. Rev. Lett. 82, 1879 (1999).

${ }^{14}$ K. Raghavachari, Q. Fu, G. Chen, L. Li, C. H. Li, D. C. Law, and R. F. Hicks, J. Am. Chem. Soc. 124, 15119 (2002).

${ }^{15}$ S. Nannarone and M. Pedio, Surf. Sci. Rep. 51, 1 (2003).

${ }^{16}$ O. Pluchery, J. Eng Jr., R. L. Opila, and Y. J. Chabal, Surf. Sci.
502-503, 75 (2002).

${ }^{17}$ O. Pluchery, Y. J. Chabal, and R. L. Opila, J. Appl. Phys. 94, 2707 (2003).

${ }^{18} \mathrm{~V}$. Swaminathan and A. T. Macrander, Material Aspects of GaAs and InP Based Structures (Prentice Hall, Englewood Cliffs, NJ, 1991).

${ }^{19}$ J. Fritsch, A. Eckert, P. Pavone, and U. Schroeder, J. Phys.: Condens. Matter 7, 7717 (1995).

${ }^{20}$ M. D. McCluskey and E. E. Haller, Semicond. Semimetals 61, 373 (1999).

${ }^{21}$ Y. J. Chabal, in Handbook of Vibrational Spectroscopy (Wiley, New York, 2001), p. 1117.

${ }^{22}$ M. D. McCluskey and E. E. Haller, Semicond. Semimetals 61, 373 (1999).

${ }^{23}$ D. W. Fischer, M. O. Manasreh, and F. Maotus, J. Appl. Phys. 71, 4805 (1992).

${ }^{24}$ D. W. Fischer, M. O. Manasreh, D. N. Talwar, and F. Maotus, J. Appl. Phys. 73, 78 (1993).

${ }^{25}$ D. W. Fischer, M. O. Manasreh, and F. Maotus, Semicond. Sci. Technol. 9, 1 (1994).

${ }^{26}$ V. Riede, H. Sobotta, H. Neumann, C. Ascheron, C. Neelmeijer, and A. Schindler, Phys. Status Solidi A 116, K147 (1989).

${ }^{27}$ C. Asheron, Phys. Status Solidi A 124, 11 (1991).

${ }^{28}$ Q. Fu, E. Negro, G. Chen, D. C. Law, C. H. Li, R. F. Hicks, and K. Raghavachari, Phys. Rev. B 65, 075318 (2002).

${ }^{29}$ G. Dlubek, C. Ascheron, R. Krause, H. Erhard, and D. Klimm, Phys. Status Solidi A 106, 81 (1988). 